KYUNGPOOK Math. J. 49(2009), 1-6

\title{
On Self-commutator Approximants
}

\author{
Bhagwati Prashad DugGaL \\ 8 Redwood Grove, Northfield Avenue Ealing, London W5 4SZ, United Kingdom \\ e-mail : bpduggal@yahoo.co.uk
}

Abstract. Let $B(\mathcal{X})$ denote the algebra of operators on a complex Banach space $\mathcal{X}, H(\mathcal{X})=\{h \in B(\mathcal{X}): h$ is hermitian $\}$, and $J(\mathcal{X})=\left\{x \in B(\mathcal{X}): x=x_{1}+\right.$ $i x_{2}, x_{1}$ and $\left.x_{2} \in H(\mathcal{X})\right\}$. Let $\delta_{a} \in B(B(\mathcal{X}))$ denote the derivation $\delta_{a}(x)=a x-x a$. If $J(\mathcal{X})$ is an algebra and $\delta_{a}^{-1}(0) \subseteq \delta_{a^{*}}^{-1}(0)$ for some $a \in J(\mathcal{X})$, then $\|a\| \leq\left\|a-\left(x^{*} x-x x^{*}\right)\right\|$ for all $x \in J(\mathcal{X}) \cap \delta_{a}^{-1}(0)$. The cases $J(\mathcal{X})=B(\mathcal{H})$, the algebra of operators on a complex Hilbert space, and $J(\mathcal{X})=\mathcal{C}_{p}$, the von Neumann-Schatten $p$-class, are considered.

\section{Introduction}

An element $h \in B(\mathcal{X}), B(\mathcal{X})=$ the algebra of (bounded linear) operators on a complex Banach space $\mathcal{X}$, is hermitian if the the algebra numerical range $V(B(\mathcal{X}), h)=\left\{f(h): f \in B(\mathcal{X})^{*}, f(I)=1=\|f\|\right\}$ is a subset of the set of reals [3, Page 8]. Let

$$
H(\mathcal{X})=\{h \in B(\mathcal{X}): h \text { is hermitian }\}
$$

and let

$$
J(\mathcal{X})=\left\{x \in B(\mathcal{X}): x=x_{1}+i x_{2}, x_{1} \text { and } x_{2} \in H(\mathcal{X})\right\} .
$$

Then each $x \in J(\mathcal{X})$ has a unique representation $x=x_{1}+i x_{2}, x_{1}$ and $x_{2} \in H(\mathcal{X})$, and we may define a mapping $x \longrightarrow x^{*}$ from $J(\mathcal{X})$ into itself by $x^{*}=x_{1}-i x_{2}$ $\left(=\left(x_{1}+i x_{2}\right)^{*}\right): J(\mathcal{X})$ with the operator norm $\|$.$\| of B(\mathcal{X})$ is a complex Banach space such that $*$ is a continuous linear involution on $J(\mathcal{X})[3$, Lemma 8 , Page 50]. Recall that an operator $a \in B(\mathcal{X})$ is normal if $a=a_{1}+i a_{2} \in J(\mathcal{X})$ and $\left[a_{1}, a_{2}\right]=a_{1} a_{2}-a_{2} a_{1}=0$. We say that an operator $a \in J(\mathcal{X})$ satisfies the $P F$ property, short for the Putnam-Fuglede property, if $a^{-1}(0) \subseteq a^{*-1}(0)$. Normal operators satisfy the PF-property: if $a=a_{1}+i a_{2}$ is normal, then $a x=0$ implies $a_{1} x=a_{2} x=0 \Longrightarrow a^{*} x=0$ [4, Page 124].

Let $\delta_{a} \in B(B(\mathcal{X}))$ denote the derivation $\delta_{a}(x)=a x-x a=\left(L_{a}-R_{a}\right) x$, where $L_{a}$ and $R_{a}$ denote, respectively, the operators of left multiplication and right multiplication by $a$. If $a \in H(\mathcal{X})$, then $L_{a}, R_{a}$ and $L_{a}-R_{a} \in H(\mathcal{X})$. Evidently, if $a=a_{1}+i a_{2}$, then $\delta_{a}=\delta_{a_{1}}+i \delta_{a_{2}}$, where $\left[\delta_{a_{1}}, \delta_{a_{2}}\right]=0$ whenever $\left[a_{1}, a_{2}\right]=0$.

Received 23 October 2007; revised 17 March 2008; accepted 17 March 2008.

2000 Mathematics Subject Classification: 47B47, 47B10, 47A30, $47 \mathrm{~B} 48$.

Key words and phrases: Banach space, von Neumann-Schatten $p$-class, derivation, kernel-range orthogonality, self-commutator. 
Hence, if $a$ is normal then $\delta_{a}$ is normal, and this by [12, Corollary 8] implies that

$$
-2 \sqrt{\left\|\delta_{a}(x)\right\|\|\| y \|}+\|x\| \leq\left\|x-\delta_{a}(y)\right\|
$$

for all $x, y \in B(\mathcal{X})$. In particular, if $x \in \delta_{a}{ }^{-1}(0)$, then (for all $y \in B(\mathcal{X})$ )

$$
\|x\| \leq\left\|x-\delta_{a}(y)\right\|
$$

i.e., the kernel $\delta_{a}{ }^{-1}(0)$ of $\delta_{a}$ is orthogonal to the range $\delta_{a}(B(\mathcal{X}))$ of $\delta_{a}$ in the sense of G. Birkhoff and R. C. James [9, page 93]. Kernel-range inequalities of type (1), especially in the setting of the algebra $B(\mathcal{H})$ (of operators on a complex Hilbert space $\mathcal{H})$ and the von Neumann-Schatten $p$-classes $\mathcal{C}_{p}=\mathcal{C}_{p}(\mathcal{H})(\mathcal{H}$ separable, $1 \leq$ $p<\infty)$ have been considered by a number of authors (see [2], [6], [7], [11], [12], [14] for further references). In this paper we look at the equation $\delta_{a}(x)=0$ from the view point that $x \in \delta_{a}^{-1}(0) \Longleftrightarrow a \in \delta_{x}^{-1}(0)$, and prove some results on self-commutator approximants of the type recently proved by P. J. Maher [13] (for self-adjoint $a$ and $\left.x \in \delta_{a}{ }^{-1}(0)\right)$. Assuming that $J(\mathcal{X})$ is an algebra (in particular, $J(\mathcal{X})=B(\mathcal{H})$ or $J(\mathcal{X})=\mathcal{C}_{p}$ for some $\left.1 \leq p<\infty\right)$ and the $\mathrm{PF}$-property that $\delta_{a}{ }^{-1}(0) \subseteq \delta_{a^{*}}^{-1}(0)$ for $a \in J(\mathcal{X})$, we prove that

$$
\|a\| \leq\left\|a-\left[x^{*}, x\right]\right\|
$$

for all $x \in J(\mathcal{X}) \cap \delta_{a}{ }^{-1}(0)$. In the case in which $1<p<\infty, \delta_{a}{ }^{-1}(0) \subseteq \delta_{a^{*}}^{-1}(0)$ and $a \in \mathcal{C}_{p}$, it is proved that

$$
\|a\|_{p} \leq \min \left\{\left\|a-\delta_{x_{1}}(y)\right\|_{p},\left\|a-\delta_{x_{2}}(y)\right\|_{p}\right\}
$$

for all $x=x_{1}+i x_{2}$ and $y \in B(\mathcal{H})$ such that $\delta_{x_{j}}(y) \in \mathcal{C}_{p}(j=1,2)$ if and only if $x \in \delta_{a}{ }^{-1}(0)$. We also prove that inequality (2) holds for essentially normal operators $x \in B(\mathcal{H}) \cap \delta_{a}^{-1}(0)$ such that $\|a\|$ equals the essential norm $\|a\|_{e}$ of $a$.

\section{Results}

Evidently, $x \in \delta_{a}^{-1}(0) \Longleftrightarrow a \in \delta_{x}^{-1}(0)$ for all $a, x \in B(\mathcal{X})$. Since $h \in H(\mathcal{X})$ does not (in general) imply that $h^{2} \in H(\mathcal{X})$ [3, Example 1, Page 58], $J(\mathcal{X})$ is not (in general) a subalgebra of $B(\mathcal{X})$. If however $J(\mathcal{X})$ is an algebra, then $h, k \in$ $H(\mathcal{X})$ implies that $h^{2}$ and $h k+k h \in H(\mathcal{X})$ [3, Theorem 3, Page 59]. Recall that $i\left(a_{1} a_{2}-a_{2} a_{1}\right) \in H(\mathcal{X})$ whenever $a_{1}, a_{2} \in H(\mathcal{X})[3$, Lemma 4, Page 47]. Let $a=a_{1}+i a_{2}$ and $b=b_{1}+i b_{2} \in J(\mathcal{X})$, and assume that $J(\mathcal{X})$ is an algebra. Then both

$$
a b+b^{*} a^{*}=\left\{\left(a_{1} b_{1}+b_{1} a_{1}\right)-\left(a_{2} b_{2}+b_{2} a_{2}\right)\right\}+i\left\{\left(a_{2} b_{1}-b_{1} a_{2}\right)+\left(a_{1} b_{2}-b_{2} a_{1}\right)\right\}
$$

and

$$
i\left(a b-b^{*} a^{*}\right)=i\left\{\left(a_{1} b_{1}-b_{1} a_{1}\right)-\left(a_{2} b_{2}-b_{2} a_{2}\right)\right\}-\left\{\left(\left(a_{2} b_{1}+b_{1} a_{2}\right)+\left(a_{1} b_{2}+b_{2} a_{1}\right)\right\}\right.
$$


are in $H(\mathcal{X})$. Hence

$$
(a b)^{*}=\frac{1}{2}\left(a b+b^{*} a^{*}\right)+\frac{i}{2}\left(a b-b^{*} a^{*}\right)=b^{*} a^{*}
$$

Theorem 2.1. If $J(\mathcal{X})$ is an algebra and $\delta_{a}^{-1}(0) \subseteq \delta_{a^{*}}^{-1}(0)$ for some a $\in J(\mathcal{X})$, then $\|a\| \leq\left\|a-\left[x^{*}, x\right]\right\|$ for all $x \in J(\mathcal{X}) \cap \delta_{a}{ }^{-1}(0)$.

Proof. The hypotheses $J(\mathcal{X})$ is an algebra and $\delta_{a}{ }^{-1}(0) \subseteq \delta_{a^{*}}^{-1}(0)$ imply that $\delta_{x}(a)=$ $\delta_{x^{*}}(a)=0$ for every $x \in J(\mathcal{X}) \cap \delta_{a}^{-1}(0)$. Hence, upon letting $x=x_{1}+i x_{2}$, $\delta_{x_{1}}(a)=\delta_{x_{2}}(a)=0$. Since $x_{j} \in H(\mathcal{X}), j=1,2$, it follows that

$$
\|a\| \leq \min \left\{\left\|a-\delta_{x_{1}}(y)\right\|,\left\|a-\delta_{x_{2}}(y)\right\|\right\}
$$

for all $y \in J(\mathcal{X})\left[12\right.$, Corollary 8]. Choose $y=2 i x_{2}\left(\right.$ in $\left.\delta_{x_{1}}(y)\right)$; then $\delta_{x_{1}}(y)=\left[x^{*}, x\right]$ and $\|a\| \leq\left\|a-\left[x^{*}, x\right]\right\|$ for all $x \in J(\mathcal{X}) \cap \delta_{a}{ }^{-1}(0)$.

The following corollary is immediate from Theorem 2.1.

Corollary 2.2. If $a \in B(\mathcal{H})$ is such that $\delta_{a}^{-1}(0) \subseteq \delta_{a^{*}}^{-1}(0)$, then $\|a\| \leq\left\|a-\left[x^{*}, x\right]\right\|$ for all $x \in B(\mathcal{H}) \cap \delta_{a}^{-1}(0)$.

An operator $a \in B(\mathcal{H})$ is essentially normal if $\pi(a)$ is normal, where $\pi$ : $B(\mathcal{H}) \longrightarrow B(\mathcal{H}) / \mathcal{K}(\mathcal{H})$ is the Calkin map. (Equivalently, $a$ is essentially normal if $\pi\left(\left[a^{*}, a\right]\right)=0$.) For essentially normal $x \in \delta_{a}^{-1}(0)$, we have the following.

Theorem 2.3. If $x \in \delta_{a}^{-1}(0) \cap B(\mathcal{H})$ is essentially normal, then $\|\pi(a)\| \leq$ $\left\|a-\left[x^{*}, x\right]\right\|$.

Proof. If $x \in \delta_{a}^{-1}(0)$, then $\pi(a) \in \delta_{\pi(x)}^{-1}(0)$. Since $B(\mathcal{H}) / \mathcal{K}(\mathcal{H})$ is a $C^{*}$-algebra, there exists a Hilbert space $\mathcal{H}_{0}$ and a $*$-isometric isomorphism $\psi: B(\mathcal{H}) / \mathcal{K}(\mathcal{H}) \longrightarrow$ $B\left(\mathcal{H}_{0}\right)$ such that $x_{0}=\psi(\pi(x))$ is a normal element of $B\left(\mathcal{H}_{0}\right)$. Letting $a_{0}=\psi(\pi(a))$, it follows that

$$
\|\pi(a)\|=\left\|a_{0}\right\| \leq \| a_{0}-\delta_{x_{0}}\left(\psi(\pi(y))\|=\| \pi\left(a-\delta_{x}(y)\right)\|\leq\| a-\delta_{x}(y) \|\right.
$$

for all $y \in B(\mathcal{H})$. Choose $y=-x^{*}$.

In general, $\|\pi(a)\| \neq\|a\|$. However, if $a \in B(\mathcal{H})$ is hyponormal (i.e., $\left|a^{*}\right|^{2} \leq$ $\left.|a|^{2}\right)$, or normaloid $(\|a\|$ equals the spectral radius of $a)$ and without eigen-values of finite multiplicity, then $\|\pi(a)\|=\|a\|$ (see [8, Page 1730]): for such $a \in B(\mathcal{H})$, $\|a\| \leq\left\|a-\left[x^{*}, x\right]\right\|$.

A version of Theorems 2.1 has been proved by Maher [13, Theorems 4.1(a) and $4.2]$ for the von Neumann-Schatten $p$-classes $\left(\mathcal{C}_{p},\|.\|_{p}\right) ; 1 \leq p<\infty$. Observe from the proof of Theorem 2.1 that if $\delta_{a}^{-1}(0) \subseteq \delta_{a^{*}}^{-1}(0)$, then $\|a\|_{p} \leq\left\|a-\left[x^{*}, x\right]\right\|_{p}$ for all $a \in \mathcal{C}_{p}$ and $x \in \delta_{a}{ }^{-1}(0)$ such that $\left[x^{*}, x\right] \in \mathcal{C}_{p}$. The following theorem proves that the condition $x=x_{1}+i x_{2} \in \delta_{a}{ }^{-1}(0)$ is necessary for $\|a\|_{p} \leq \min \{\| a-$ 
$\left.\delta_{x_{1}}(y)\left\|_{p},\right\| a-\delta_{x_{2}}(y) \|_{p}\right\}$ in the case in which $1<p<\infty$. But before that we introduce some terminology. If $(\mathcal{V},\|\|$.$) is a Banach space, then \|$.$\| is said to be$ Gateaux-differentiable at a non-zero $x \in \mathcal{V}$ if

$$
\lim _{t \longrightarrow 0} \frac{\|x+t y\|-\|x\|}{t}=\operatorname{Re} D_{x}(y)
$$

exists for all $y \in \mathcal{V}$. Here $t \in \mathcal{R}$ (= the set of reals), Re denotes the real part and $D_{x}$ is the unique support functional in the dual space $\mathcal{V}^{*}$ such that $\left\|D_{x}\right\|=1$ and $\left\|D_{x}(x)\right\|=\|x\|$. The Gateaux-differentiability of $\|$.$\| at x$ implies that $x$ is a smooth point of the sphere with radius $\|x\|$. If an $a \in \mathcal{C}_{p}, 1<p<\infty$, has the polar decomposition $a=u|a|$, then $|a|^{p-1} u^{*} \in \mathcal{C}_{p^{\prime}}, \frac{1}{p}+\frac{1}{p^{\prime}}=1$, and $D_{a}(y)=\operatorname{tr}\left\{|a|^{p-1} u^{*} y / \|\left. a\right|_{p} ^{p-1}\right.$ ) for every $y \in \mathcal{C}_{p}$ [1, Theorem 2.3]. (As usual, tr denotes the trace functional.) Recall from [10] that if $a, b \in \mathcal{V}$ and $a$ is a smooth point of $\mathcal{V}$, then $\|a\| \leq\|a+t b\|$ for all complex $t$ if and only if $D_{a}(b)=0$.

Theorem 2.4. If $\delta_{a}^{-1}(0) \subseteq \delta_{a^{*}}^{-1}(0)$ then

$$
\|a\|_{p} \leq \min \left\{\left\|a-\delta_{x_{1}}(y)\right\|_{p},\left\|a-\delta_{x_{2}}(y)\right\|_{p}\right\}
$$

for all $a \in \mathcal{C}_{p}$ and $y \in B(\mathcal{H})$ such that $\delta_{x_{j}}(y) \in \mathcal{C}_{p}, j=1,2$ and $1<p<\infty$, if and only if $x=x_{1}+i x_{2} \in \delta_{a}{ }^{-1}(0)$.

Proof. The 'if part' being evident (from $\delta_{a}(x)=0 \Longrightarrow \delta_{x_{1}}(a)=\delta_{x_{2}}(a)=0$ ), we prove the 'only if' part. Recall that $\mathcal{C}_{p}, 1<p<\infty$, is uniformly convex; hence operators $a \in \mathcal{C}_{p}$ are smooth points of $\mathcal{C}_{p}$. Let $a$ have the polar decomposition $a=u|a|$. Then, [10], the inequality of the statement of the theorem holds for all $y \in B(\mathcal{H})$ such that $\delta_{x_{j}}(y) \in \mathcal{C}_{p}, j=1,2$, if and only if the support functional $D_{a}\left(\delta_{x_{j}}(y)\right)=\operatorname{tr}\left(|a|^{p-1} u^{*} \delta_{x_{j}}(y) /\|a\|_{p}^{p-1}\right)=0$. Set $|a|^{p-1} u^{*}=\tilde{a}$; then $\tilde{a} \in \mathcal{C}_{p^{\prime}}$, $\frac{1}{p}+\frac{1}{p^{\prime}}=1$. Choose $y$ to be the rank one operator $y=e \otimes f$ for some $e, f \in \mathcal{H}$. Then $\delta_{x_{j}}(y) \in \mathcal{C}_{p}$ and

$$
\begin{aligned}
\operatorname{tr}\left(\tilde{a} \delta_{x_{j}}(y)\right) & =\operatorname{tr}\left(\tilde{a}\left(x_{j} y-y x_{j}\right)\right)=\operatorname{tr}\left(\left(\tilde{a} x_{j}-x_{j} \tilde{a}\right) y\right) \\
& =\operatorname{tr}\left(\delta_{\tilde{a}}\left(x_{j}\right) e \otimes f\right)=\left(\delta_{\tilde{a}}\left(x_{j}\right) e, f\right)=0
\end{aligned}
$$

for all $e, f \in \mathcal{H}$. Hence $\delta_{\tilde{a}}\left(x_{j}\right)=0 ; j=1,2$. The operator $x_{j}$ being self-adjoint

$$
u|a|^{p-1} x_{j}=x_{j} u|a|^{p-1} \Longrightarrow|a|^{2(p-1)} x_{j}=|a|^{p-1} u^{*} x_{j} u|a|^{p-1}=x_{j}|a|^{2(p-1)} .
$$

Hence $\left[x_{j},|a|\right]=0$. Since $\tilde{a} x_{j}=x_{j} \tilde{a}$ implies $\left.\left[x_{j}, u\right]\right|_{\overline{\operatorname{ran}|a|^{p-1}}}=0$, it follows that

$$
a x_{j}=u|a| x_{j}=u x_{j}|a|=x_{j} u|a|=x_{j} a .
$$

Hence $\delta_{a}\left(x_{1}\right)+i \delta_{a}\left(x_{2}\right)=\delta_{a}(x)=0$.

A stronger result is possible in the case in which $p=2$. 
Corollary 2.5. If $a \in \mathcal{C}_{2}$, then

$$
\left\|a+\delta_{x_{j}}(y)\right\|_{2}^{2}=\|a\|_{2}^{2}+\left\|\delta_{x_{j}}(y)\right\|_{2}^{2}=\left\|a^{*}+\delta_{x_{j}}(y)\right\|_{2}^{2}, \quad j=1,2,
$$

for all $y \in \mathcal{C}_{2}$ if and only if $x=x_{1}+i x_{2} \in \delta_{a}^{-1}(0) \cap \delta_{a^{*}}^{-1}(0)$.

Proof. $\mathcal{C}_{2}$ has a Hilbert space structure with inner product $(s, t)=\operatorname{tr}\left(t^{*} s\right)$. Since

$$
\begin{aligned}
& \left\|a+\delta_{x_{j}}(y)\right\|_{2}^{2}=\|a\|_{2}^{2}+\left\|\delta_{x_{j}}(y)\right\|_{2}^{2}+2 \operatorname{Re}\left(\delta_{x_{j}}(y), a\right), \\
& \left\|a^{*}+\delta_{x_{j}}(y)\right\|_{2}^{2}=\|a\|_{2}^{2}+\left\|\delta_{x_{j}}(y)\right\|_{2}^{2}+2 \operatorname{Re}\left(a, \delta_{x_{j}}^{*}(y)\right)=\|a\|_{2}^{2}+\left\|\delta_{x_{j}}(y)\right\|_{2}^{2}+2 \operatorname{Re}\left(a, \delta_{x_{j}}(y)\right), \\
& \left(\delta_{x_{j}}(y), a\right)=\operatorname{tr}\left(a^{*} \delta_{x_{j}}(y)\right)=\operatorname{tr}\left(\delta_{a^{*}}\left(x_{j}\right) y\right)=\operatorname{tr}\left(\delta_{a}^{*}\left(x_{j}\right) y\right),
\end{aligned}
$$

and

$$
\left(a, \delta_{x_{j}}(y)\right)=\operatorname{tr}\left(\delta_{x_{j}}(y) a\right)=\operatorname{tr}\left(\delta_{a}\left(x_{j}\right) y\right),
$$

it follows that if $x \in \delta_{a}{ }^{-1}(0) \cap \delta_{a^{*}}^{-1}(0)$, then $\left\|a+\delta_{x_{j}}(y)\right\|_{2}^{2}=\|a\|_{2}^{2}+\left\|\delta_{x_{j}}(y)\right\|_{2}^{2}=\| a^{*}+$ $\delta_{x_{j}}(y) \|_{2}^{2}$ for all $y \in \mathcal{C}_{2}$. Conversely, if this equality is satisfied, then the argument of the proof Theorem 2.4 (with $p=2$ ) applied to the inequalities $\|a\|_{2} \leq\left\|a-\delta_{x_{j}}(y)\right\|_{2}$ and $\left\|a^{*}\right\|_{2} \leq\left\|a^{*}-\delta_{x_{j}}(y)\right\|_{2}$ implies that $x_{j}$, and so also $x, \in \delta_{a}^{-1}(0) \cap \delta_{a^{*}}^{-1}(0)$.

The elementary operator $\triangle_{a}(x)=a x a-x$. We close this note with a remark on the elementary operator $\triangle_{a}$. If $a \in B(\mathcal{X})$ is a contraction, then $L_{a} R_{a}$ is a contraction. Hence

$$
\left.V(B(B(\mathcal{X}))), L_{a} R_{a}\right) \subseteq\{\lambda \in C:|\lambda| \leq 1\}
$$

and

$$
V\left(B(B(\mathcal{X})), \triangle_{a}\right)=V\left(B(B(\mathcal{X})), L_{a} R_{a}-I\right) \subseteq\{\lambda \in C:|\lambda+1| \leq 1\}
$$

[5, Proposition 4, Page 52]. (Here $C$ denotes the complex plane.) This implies that the operator $\triangle_{a}$ is dissipative [3, Page 30], and hence

$$
\|x\| \leq\left\|x-\triangle_{a}(y)\right\|
$$

for all $x \in \triangle_{a}^{-1}(0)$ and $y \in B(\mathcal{X})\left[12\right.$, Theorem 7]. Although $\triangle_{a}$ may not be normal even for normal $a \in B(\mathcal{X})$, see [7, Example 2.1], a number of kernel-range orthogonality results for the elementary operator $\triangle_{a} \in B(B(\mathcal{H}))$ and $\triangle_{a} \in B\left(\mathcal{C}_{p}\right)$ are to be found in the extant literature; see for example [6], [7], [11], [14]. Seemingly, self-commutator approximant inequalities of the type (2) are not possible for $\triangle_{a}$. However, one does have the following interesting result.

Theorem 2.6. Assume that $\triangle_{a}^{-1}(0) \subseteq \triangle_{a^{*}}^{-1}(0)$. If $a \in B(\mathcal{H})$ (resp., $a \in \mathcal{C}_{p}$ ), then $\|a\| \leq \| a-\left[|x|,\left|x^{*}\right|\right]||$ for all $x \in B(\mathcal{H}) \cap \triangle_{a}^{-1}(0)$ (resp., $\|a\|_{p} \leq\left\|a-\left[|x|,\left|x^{*}\right|\right]\right\|_{p}$ for all $x \in \mathcal{C}_{p} \cap \triangle_{a}^{-1}(0)$ ). 
Proof. If $x \in \triangle_{a}^{-1}(0)$, then $a x a=x$ and $a^{*} x a^{*}=x\left(\Longleftrightarrow a x^{*} a=x^{*}\right)$. Since

$$
a x^{*} x=\left(a x^{*}\right) a x a=\left(a x^{*} a\right) x a=x^{*} x a,
$$

$[a,|x|]=0$. Hence $\delta_{|x|}(a)=0$, which, since $|x| \geq 0$, implies that $\|a\| \leq\left\|a-\delta_{|x|}(y)\right\|$ for all $y \in B(\mathcal{H})$ (resp., $\|a\|_{p} \leq\left\|a-\delta_{|x|}(y)\right\|_{p}$ for all $y \in B(\mathcal{H})$ such that $\left.\delta_{|x|}(y) \in \mathcal{C}_{p}\right)$. Choose $y=\left|x^{*}\right|$. Since $x \in \mathcal{C}_{p}$ implies $|x| \in \mathcal{C}_{p}$, the proof is complete.

\section{References}

[1] T. A. Abatzoglou, Norm derivatives on spaces of operators, Math. Ann., 239(1979), 129-135.

[2] J. Anderson and C. Foiaş, Properties which normal operators share with normal derivations and related operators, Pac. J. Math., 61(1975), 313-325.

[3] F. F. Bonsall and J. Duncan, Numerical ranges I, Lond. Math. Soc. Lecture Notes Series, 2(1971).

[4] F. F. Bonsall and J. Duncan, Numerical ranges II, Lond. Math. Soc. Lecture Notes Series, 10(1973).

[5] F. F. Bonsall and J. Duncan, Complete Normed Algebras, Ergebnisse der Math. und ihrer Grenzgebiete, Band 80, Springer-Verlag, (1973).

[6] B. P. Duggal, Range-kernel orthogonality of the elementary operator $X \longrightarrow$ $\sum_{i=1}^{n} A_{i} X B_{i}-X$, Lin. Alg. Appl., 337(2001), 79-86.

[7] B. P. Duggal, Subspace gaps and range-kernel orthogonality of an elementary operator, Lin. Alg. Appl., 383(2004), 93-106.

[8] B. P. Duggal, A perturbed elementary operator and range-kernel orthogonality, Proc. Amer. Math. Soc., 134(2006), 1727-1734.

[9] N. Dunford, J. T. Schwartz, Linear Operators, Part I, Interscience, New York, (1964).

[10] R. C. James, Orthogonality and linear functionals in normed linear spaces, Trans. Amer. Math. Soc., 61(1947), 265-292.

[11] Dragoljub Kečkić, Orthogonality of the range and the kernel of some elementary operators, Proc. Amer. Math. Soc., 128(2000), 3369-3377.

[12] Yuan-Chuan Li and Sen-Yen Shaw, An absolute ergodic theorem and some inequalities for operators on Banach spaces, Proc. Amer. Math. Soc., 125(1997), 111-119.

[13] P. J. Maher, Self-commutator approximants, Proc. Amer. Math. Soc., 134(2006), 157-165.

[14] Aleksej Turnšek, Orthogonality in $\mathcal{C}_{p}$ classes, Mh. Math., 132(2001), 349-354. 